after the first iv infusion. (Fayad MN, Choueiri R, Mikati M. Landau-Kleffner syndrome: consistent response to repeated intravenous g-globulin doses: a case report. Epilepsia April 1997;38:489-494). (Reprints: Dr MN Fayad, AUB NY Office, 850 Third Ave (18th floor), New York, NY 10022).

COMMENT. Landau-Kleffner syndrome (LKS) has multiple etiologies, including meningitis, demyelination, arteritis, and cerebral neoplasms. Trials of AEDs and corticosteroids have been disappointing, providing at best some temporary relief. The present case report with dramatic response to immunotherapy suggests a postencephalitic autoimmune abnormality, and further studies are indicated. The late Dr Frank Morrell advocated subpial intracortical transection in selected cases of LKS. (see Progress in Pediatric Neurology III, 1997;p86).

\title{
OCCIPITAL SEIZURES V. MIGRAINE AURA
}

Three children, ages 13 to 17 years, with occipital seizures resembling the visual aura of migraine are reported from St Thomas' Hospital, London, England. Case 1, a 14-year-old boy with weekly episodes of visual hallucinations beginning at age 8 , first complained of concentric spherical rings of red and yellow moving from left to right visual field, without impaired consciousness, convulsion, or headache. After age 10 years, the visual hallucinations were followed by left-sided tonic deviation of the head and clonic movements of left face and arm, accompanied by loss of consciousness, post-ictal sleep and headache. Interictal EEGs and MRI were normal. Carbamazepine started at age 11 years prevented further attacks. Cases 2 and 3 also had visual hallucinations accompanied by headache and either loss of posture or convulsive movements; MRIs were normal, EEGs showed no epileptiform activity, and carbamazepine controlled attacks in one. The other patient refused treatment and attacks continued. (Panayiotopoulos CP, Sharoqi IA, Agathonikou A. Occipital seizures imitating migraine aura. IR Soc Med May 1997;90:255-257). (Respond: Dr Panayiotopoulos, Dept Clinical Neurophysiology and Epilepsies, St Thomas' Hospital, London SE1 7EH, England).

COMMENT. The interpretation of the visual hallucinations in these three children as epileptic events, and differing from migraine, is based on clinical manifestations, including the brevity of the symptom, multicolored patterns rather than black and white, onset on the same side, lack of photophobia, mild post-ictal headache, and response to antiepileptic medication. The lack of epileptiform activity on interictal EEGs is troublesome, and a positive video-EEG recording during an attack would have been more convincing. The authors stress the need to evaluate visual hallucinations both quantitatively and qualitatively to distinguish epilepsy, and especially benign childhood occipital seizures, from migraine phenomena.

\section{CARBAMAZEPINE-INDUCED MOTOR IMPAIRMENTS}

Treatment with carbamazepine (CBZ) in therapeutic levels was associated with motor impairments in 19 children with epilepsy, studied during and 6 months after treatment was withdrawn at Huddinge University Hospital, Sweden. On a Bruininks-Oseretsky standardized test of gross and finemotor proficiency, the drug-free evaluation showed significant improvements in response speed, fine-motor function, and total test battery scores. In another group of 12 children tested during treatment with CBZ, visual-motor control was impaired on the second test at a 6 month interval. (Braathen $G$, von 
Bahr L, Theorell K. Motor impairments in children with epilepsy treated with carbamazepine. Acta Paediatr April 1997;86:372-376). (Respond: Dr G Braathen, Department of Paediatrics, Huddinge University Hospital, S-141 86 Huddinge, Sweden).

COMMENT. Fine motor coordination impairments in children with epilepsy treated with carbamazepine may be explained by effects of the antiepileptic drug.

Cerebellar adverse effects of carbamazepine are reported in 9 (35\%) of 26 young adults with chronic focal epilepsy treated at the Klinik Mara 1, Epilepsie-Zentrum Bethel, Bielefeld, Germany. (Specht U, May TW, Rohde M et al. Cerebellar atrophy decreases the threshold of carbamazepine toxicity in patients with chronic focal epilepsy. Arch Neurol April 1997;54:427-431). Serum concentrations of $\mathrm{CBZ}$ at onset of ataxic side-effects were related to occurrence of cerebellar atrophy on MRI. Patients showing cerebellar atrophy developed ataxia, nystagmus, and dizziness at lower CBZ levels than those without cerebellar atrophy.

\section{EPILEPSY, ADHD, AND METHYLPHENIDATE}

The effects of methylphenidate in thirty children, ages 6 to 16 , with epilepsy and ADHD were evaluated in a 4-month period at Shaare Zedek Medical Center, Hadassah-Hebrew University, Jerusalem, Israel. Using a double-blind, crossover design, patients received EEGs, AED level determinations, and continuous-performance tasks during treatment with AEDs only, and with the addition of methylphenidate (MPH), $0.3 \mathrm{mg} / \mathrm{kg}$ each morning, or placebo. Three of 5 children with seizures had exacerbation of attacks ( 3 to 7 attacks per week) while taking MPH, while 25 without seizures continued to be seizurefree. MPH improved ADHD symptoms and continuous-performance task scores. (Gross-Tsur V, Manor O, van der Meere J, Joseph A, Shalev RS. Epilepsy and attention deficit hyperactivity disorder: Is methylphenidate safe and effective? I Pediatr April 1997;130:670-674). (Reprints: Ruth Shalev MD, Pediatric Neurobehavioral Unit, Shaare Zedek Medical Center, POB 3235, Jerusalem, Israel).

COMMENT. Methylphenidate (MPH) may be a safe and effective treatment for ADHD complicating childhood epilepsy, provided that patients are receiving antiepileptic drugs when MPH is initiated and that seizures are under satisfactory control. Patients with persisting seizures despite AEDs may have an exacerbation with MPH. Existing studies do not address the problem of patients with ADHD in whom a predisposition to seizures may be overlooked before stimulant therapy is introduced. Furthermore, the effects of Ritalin ${ }^{\circledR}$ may be different from generic MPH.

Ritalin-induced seizures in two children with ADHD. Two boys, ages 8 and 11 years, were seen in the ADD Clinic, Division of Neurology, Children's Memorial Hospital, Chicago, with a history of convulsive seizures after Ritalin had been introduced for treatment of ADHD. With the addition of carbamazepine and withdrawal or reduction in dosage of Ritalin, seizures had not recurred. (Millichap JG, Swisher CN. Manuscript in preparation).

\section{TOURETTE SYNDROME}

\section{METABOLIC ANATOMY OF TOURETTE'S SYNDROME}

A Scaled Subprofile Statistical Model (SSM) of regional metabolic covariation was employed to identify functional brain networks in 10 Tourette 